\title{
Measurements of Laser Induced Bubble Behavior in Elastic Tube and Temperature around Bubble in TUL Treatment
}

\author{
Yasuhiro Sugimoto*, Masamichi Hamamoto \\ Department of Mechanical Engineering, Kanazawa Institute of Technology, Ishikawa, Japan \\ Email: *y-sugi@neptune.kanazawa-it.ac.jp
}

How to cite this paper: Sugimoto, Y. and Hamamoto, M. (2020) Measurements of Laser Induced Bubble Behavior in Elastic Tube and Temperature around Bubble in TUL Treatment. Journal of Flow Control, Measurement \& Visualization, 8, 134-145. https://doi.org/10.4236/jfcmv.2020.83008

Received: March 26, 2020

Accepted: June 23, 2020

Published: July 20, 2020

Copyright $\odot 2020$ by author(s) and Scientific Research Publishing Inc. This work is licensed under the Creative Commons Attribution International License (CC BY 4.0).

http://creativecommons.org/licenses/by/4.0/

(c) (i) Open Access

\begin{abstract}
Transurethral ureteral lithotripsy (TUL) is a treatment that breaks stones by irradiating a pulsed laser through an optical fiber. Heat and impulsive force of the laser may affect nearby tissues during treatment. A bubble induced by the pulsed laser plays an important role in laser lithotripsy. It is important to understand effects of the bubble on the surroundings by simulating treatment in a narrow space such as in a ureter. In this study, we observe behaviors of the bubble in the narrow space inside a soft material simulating under in vivo condition. The bubble formed under various laser irradiation conditions exhibits characteristic behavior, and the surrounding elastic wall is compressed and bulged when the bubble grows and collapses. In the case of bubble formed near the elastic wall, the bubble contacts with the elastic wall during growth, and severe large deformation of the elastic wall is observed at bubble collapse. According to the temperature measurement, a temperature rise of $25^{\circ} \mathrm{C}-30^{\circ} \mathrm{C}$ occurs in the area where the bubbles are in contact. From the above, by presenting the deformation of the elastic wall and temperature increase, we can show useful information to improve the safety for treatment at narrow space.
\end{abstract}

\section{Keywords}

Ho:YAG Laser, Laser Induced Bubble, Lithotripsy, Bubble Behavior in Elastic Tube, Temperature around Bubble

\section{Introduction}

Extracorporeal shock waves, focused ultrasound, and transurethral ureteral lithotripsy (TUL) have been used effectively for treatment of calculi in the human body [1]-[10]. TUL is a treatment that uses a laser to crush stones while check- 
ing them with an endoscope. The pulsed laser is repeatedly irradiated on the affected part through an optical fiber, and calculus crushing is performed. Calculi are broken by the thermal effects of direct laser irradiation and the effects of bubbles formed. In this study, we focus on the behavior of bubbles formed during laser irradiation. There are many studies on the generation and collapse of laser-induced bubbles near rigid walls and their impact. For example, Lauterborn [11] and Vogel et al. [12] have reported many results on bubbles generated by laser focusing. Tomita et al. [13] and Lauterborn [11] have collated the erosion mark with the collapse behavior of a single bubble. Recently, Brujan et al. [14] [15] and Kobayashi et al. [16] have shown the effect on the elasticity of a wall in the vicinity of an elastic wall other than a rigid wall. Kodama et al. [17] demonstrated bubble collapse near the elastic wall and jet rush into gelatin. Furthermore, Blake et al. [18], Chahine [19], and Ogasawara et al. [20] have shown characteristic bubble collapse behaviors near rigid or elastic walls under various conditions, including bubble behavior near free surfaces.

Examples associated with impact seem to be less than bubbles produced by focused lasers though some observations have been performed on the laser-induced bubbles through the fiber studied in this study (e.g., Refs. [9] [10]). The actual treatment is performed in a narrow space inside the body, and studies on the behavior in a narrow space and near an elastic wall [14] [15] [16] [21] do not seem to be much.

The authors investigated the relationship between the bubble behavior near the rigid wall and the impact on the laser-induced bubble through the fiber, presented the conditions for the formation of the bubble that gives the optimal impact on the rigid wall, and behavior of bubble formed in a narrow space with the two flat plates [22] [23] [24]. Such growth and collapse of bubbles formed by evaporation of water at the irradiation point occur near the elastic wall even for a short time. The bubble formation/collapse is repeated at several tens to $100 \mathrm{~Hz}$ in clinical treatment, which may lead to mechanical and thermal damage to internal tissues. It is necessary to understand the behavior and bubble temperature. In particular, when hot vapor bubbles come into direct contact with living tissue, they can have a thermal effect. In this study, we observed the behavior of bubbles formed under the laser irradiation conditions used in actual treatment in a narrow cylindrical tube made by elastic walls, and investigated the behavior of bubbles and surrounding elastic walls. In addition, in order to investigate the temperature rise on the elastic wall caused by the contact of the bubble, the temperature field around the bubble in a large free space filled with water was measured using a thermocouple, albeit time-averaged.

\section{Experimental Apparatus and Procedure}

The laser device used was a laser treatment device (Lumenis, VeasaPulse 80/100W) used in medical treatment [22]. The pulse laser (Ho:YAG laser, wavelength: $2100 \mathrm{~nm}$ ) was irradiated through using a quartz glass fiber (Boston Scientific Japan, Ho:YAG \& Nd:YAG, $50 \mathrm{~W}$, fiber core diameter: $242 \mu \mathrm{m}$ ). In addi- 
tion, the end surface of the fiber is arranged perpendicularly and smoothly to its axis. The laser energy $E$ is $1.0 \mathrm{~J}$ and the pulse duration is about $0.3 \mathrm{~ms}$. Figure 1 shows an example of a laser pulse waveform at a laser energy of $1.0 \mathrm{~J}$ and a repetition frequency of $40 \mathrm{~Hz}$ measured by a photodetector (Thorlabs, DET10D/M).

Figure 2 shows the details of the test section. The fibers were placed at $X_{W}=$ $0.3,1.0$, and $2.5 \mathrm{~mm}$ from the elastic wall of a cylinder (diameter $5 \mathrm{~mm}$ ) made of soft material (gelatin) in the tube center direction. Here, the diameter of the circular tube was selected from the actual ureter (4 to $7 \mathrm{~mm}$ in diameter) and the scale of the endoscope used for the treatment. The test section was submerged in an acrylic resin water vessel filled with tap water (water temperature: Tw). In order to observe bubble behaviors, two high-speed video cameras (Camera A: NAC, GX-1 PLUS and Camera B: Photron, FASTCAM SA5) were synchronized with the sound pressure that generated by bubble is acquired by a hydrophone (B \& K, 8103) placed outside the tube. Here, the behaviors were photographed by a backlight method using a metal halide lamp (NPI, PCS-UMX350) and a plane light guide (NPI, PLG-B100X). The images were taken at 50,000 fps using camera A from the side of the tube and at 100,000 fps using camera B from the side of the tube axis. The dissolved oxygen content $\beta$ was measured using a dissolved oxygen meter (HORIBA, OM-51).

Figure 3 shows the test section used for measuring the temperature of the water around the bubbles. A thermocouple (Okazaki, T801-1) with an outer diameter of $0.06 \mathrm{~mm}$ was used for the measurement. In order to prevent the thermocouple from moving along with the bubble movement, the thermocouple and a $\varphi 1.0 \mathrm{~mm}$ piano wire were fixed with adhesive. Here, the laser energy was set to $1.0 \mathrm{~J}$. As shown in Figure 3, the fiber tip and the thermocouple tip were placed so that they coincided, and the distance $x_{t}$ from the fiber center to the thermocouple surface was set to $1.0,1.5,2.0,3.0$, or $4.0 \mathrm{~mm}$. The thermocouple was connected to a data logger (KEYENCE, NR-500 and NR-TH08) and the data was recorded on a PC. Here, the sampling frequency of the data logger is $10 \mathrm{~Hz}$, which is slower than the bubble life. For this reason, the effect of laser irradiation on water temperature rise was acquired in a time-averaged manner by measuring the temperature change during a 10 -second pulse laser irradiation at a frequency of $40 \mathrm{~Hz}$ with a $1.0 \mathrm{~J}$ pulse laser. At the same time as the temperature measurement, the behavior of bubbles was observed using camera A.

The test section is composed of gelatin (Sigma-Aldrich, G2500 Type A) made with a mass ratio of 20 mass\% to water. A uniaxial compression test based on JIS K6254 was performed on a test piece prepared from a gelatin solution, and the compression modulus was measured. Here, in order to examine the change in elastic modulus due to water absorption and temperature change of the soft material during the experiment, test pieces were used immediately after cooling was completed and left standing in water (water temperature $18^{\circ} \mathrm{C}-19^{\circ} \mathrm{C}$ ) for 30 minutes. The elastic modulus immediately after cooling is about $94 \mathrm{kPa}$, and the value after standing in water for 30 minutes is about $53 \mathrm{kPa}$. 


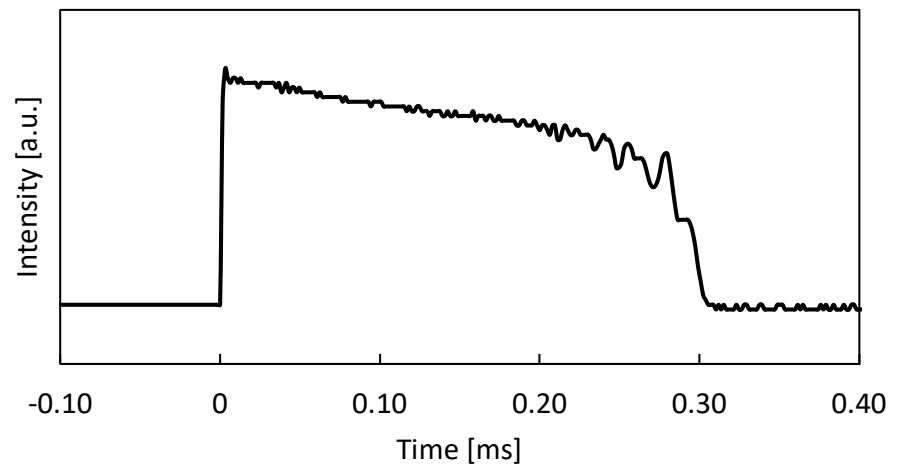

Figure 1. Temporal change of laser pulse strength.

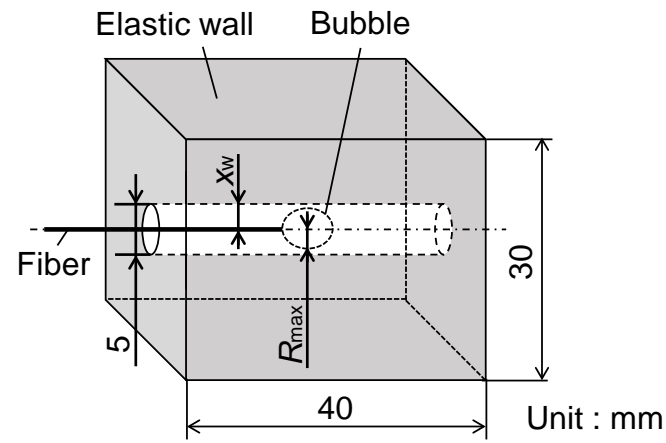

Figure 2. Details of test section.

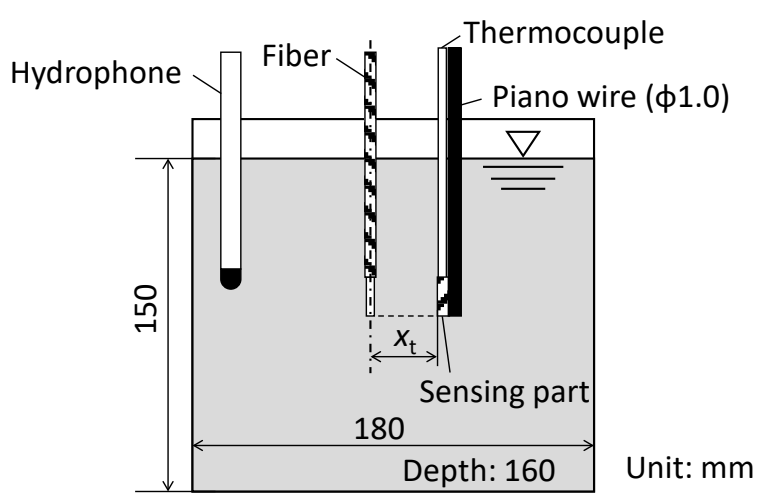

Figure 3. Test section for temperature measurement.

\section{Behavior of Bubbles and Elastic Walls in a Cylindrical Tube}

Figure 4 shows the bubble behavior (distance from the elastic wall $x_{W}=0.3,1.0$, and $2.5 \mathrm{~mm}$ ) at each fiber position in the cylindrical tube observed from two directions, the tube side direction and the tube axis direction. Here, the behavior of a single bubble formed by single laser irradiation will be described. From the obtained image, the bubble formation position was estimated as the ratio $\gamma_{w}$ ( $x_{w} / R_{\max }$ ) of the distance $x_{W}$ from the elastic wall to the center of the fiber and the distance $R_{\max }$ from the elastic wall of the largest expanding bubble. Note that the measured values of $x_{W}$ and $R_{\max }$ each include an uncertainty of about $0.05 \mathrm{~mm}$ 
due to the lens effect because the refractive index of the soft material is slightly different from that of water. The time of one frame before the formation of bubbles is defined as $t=0.00 \mathrm{~ms}$. Bubbles are formed in part A at $t=0.02 \mathrm{~ms}$ in Figure 4(a), and grow long in the laser irradiation direction (cylinder axis direction). After reaching the maximum diameter near the part $\mathrm{B}$ at $t=0.32 \mathrm{~ms}$ (the time when the laser pulse duration is almost finished), the bubbles begin to shrink by being deprived of heat by the surrounding water and condensed. The bubble is greatly deformed from the long axis direction and collapses near the fiber tip at $t=0.62 \mathrm{~ms}$ (it became the smallest diameter). Here, no large bubble splitting occurs between the parallel plates [23] [24]. Subsequently, the non-condensable gas such as air contained in the bubbles is not completely dissolved in water in a short time, and the bubbles are expanded again $(t=0.64 \mathrm{~ms})$ using the remaining fine bubbles as nuclei, and then the bubbles collapses again ( $t=0.82 \mathrm{~ms}$ ). The deformation of the elastic wall is observed with the growth and shrinkage of the bubbles.

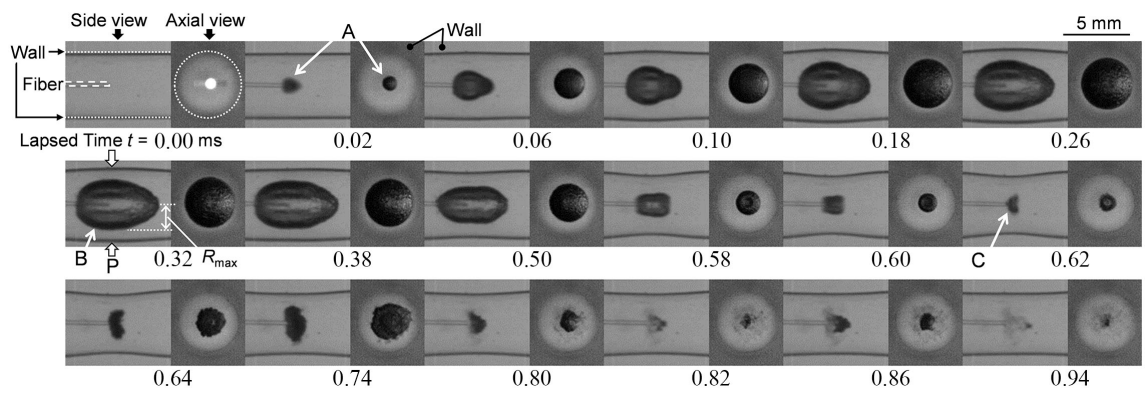

(a) $\gamma_{\mathrm{w}}=1.3, x_{\mathrm{w}}=2.5 \mathrm{~mm}$

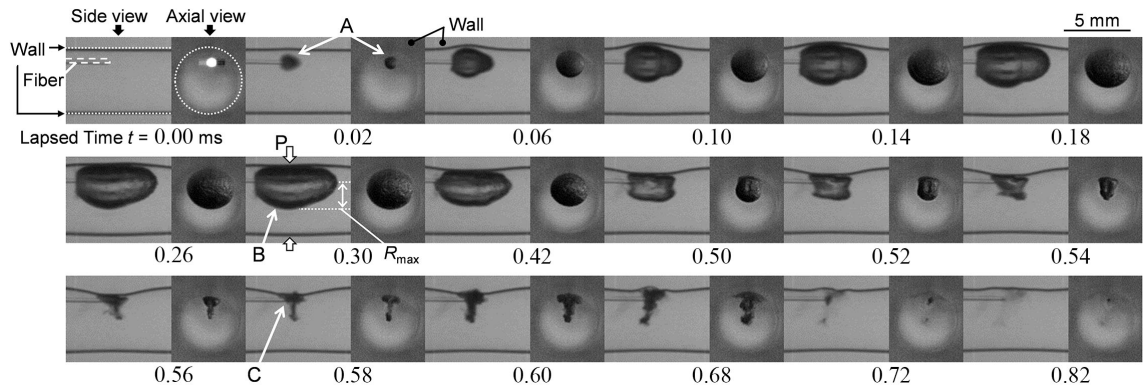

(b) $\gamma_{\mathrm{w}}=0.5, x_{\mathrm{w}}=1.0 \mathrm{~mm}$

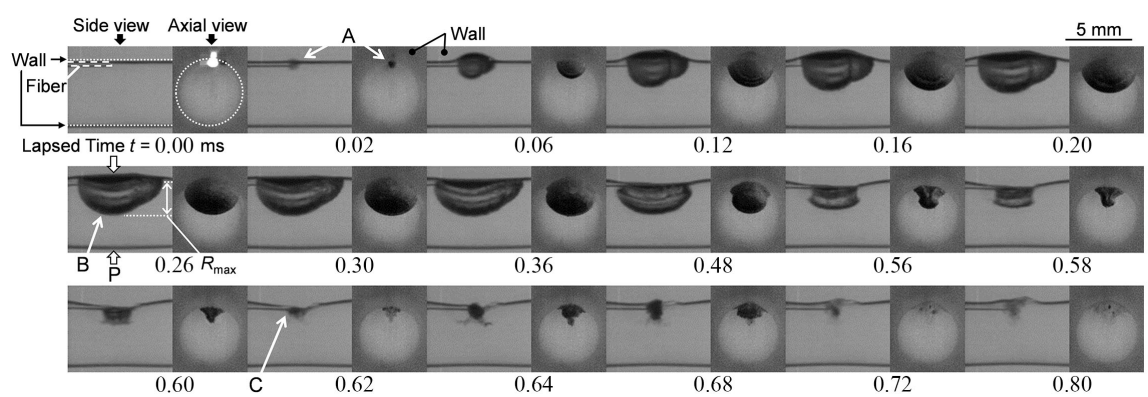

(c) $\gamma_{\mathrm{w}}=0.1, x_{\mathrm{w}}=0.3 \mathrm{~mm}$

Figure 4. Behaviors of a laser-induced bubble and an elastic wall. $T_{\mathrm{w}}=16^{\circ} \mathrm{C}-20^{\circ} \mathrm{C}, \beta=$ $7.3 \mathrm{mg} / \mathrm{L}$. 
When the fiber position is close to the elastic wall (Figure 4(b), $X_{W}=1.0 \mathrm{~mm}$ ), bubble formation is confirmed in section A when $t=0.02 \mathrm{~ms}$. Bubbles contact the elastic wall around $0.10 \mathrm{~ms}$ and grow while pushing the elastic wall. After the bubble reaches its maximum diameter at $\mathrm{B}$ at $t=0.30 \mathrm{~ms}$, it begins to shrink with large deformation of the elastic wall, and collapses on the elastic wall at $\mathrm{C}$ at $t=0.58 \mathrm{~ms}$. After that, the bubble rebounds, and when it collapses again, it is confirmed that the elastic wall deforms intricately (around $0.72 \mathrm{~ms}$ ). As shown in Figure 4(c), when the fiber position becomes closer the elastic wall, the bubble grows in contact with the elastic wall from the bubble formation in section $\mathrm{A}$ at $t=0.02 \mathrm{~ms}$. While the elastic wall returns to its original position, the bubble reaches its maximum diameter near $\mathrm{B}$ at $t=0.30 \mathrm{~ms}$. When the bubble shrinks, it deforms so as to be constricted from the longitudinal direction near the elastic wall (around $0.52 \mathrm{~ms}$ ), and collapses at the part C near $t=0.62 \mathrm{~ms}$. Complex elastic wall deformation behavior is observed by subsequent rebound and collapse behavior $(0.80 \mathrm{~ms})$.

Figure 5 shows the deformation of the bubble interface and the elastic wall. The vertical 1-pixel-wide images (about $0.06 \mathrm{~mm}$ ) at the position where the bubble width becomes maximum at the time of maximum bubble expansion (arrow $\mathrm{P}$ in Figure 4) are arranged in chronological order. When the fiber position $X_{W}=2.5 \mathrm{~mm}$, the elastic wall deforms in the direction of compression from the time of $t=0.0 \mathrm{~ms}$ during bubble growth. After the bubble reaches its maximum diameter at $t=0.32 \mathrm{~ms}$, the elastic wall deforms to be pulled by the bubble as the bubble contracts. The gap between the bubble surface and the elastic wall decreases with the growth of the bubble, but the bubble does not touch the elastic wall because the elastic wall is deformed correspondingly.

In Figure 5(b), the elastic wall shows the maximum deformation around $t=$ $0.15 \mathrm{~ms}$ before the bubble reaches the maximum expansion, and then the elastic wall begins to deform in the direction to return to the original position. After the bubble collapse (part C at $t=0.58 \mathrm{~ms}$ in Figure 4), the elastic wall deforms again in the direction of compression (around $t=0.60-0.70 \mathrm{~ms}$ ) as the bubble rebounds. Under the condition that the fiber position is closer to the elastic wall, as shown in Figure 5(c), the deformation of the elastic wall due to bubble growth is larger than in Figure 5(a) as shown in Figure 5(b) (around $t=0.15$ $\mathrm{ms}$ ). However, the bubble diameter becomes smaller due to the effect of the elastic wall compared to the condition where the fiber is farthest from the elastic wall (Figure 5(a)). It is also confirmed that the elastic wall far from the fiber is slightly deformed.

\section{Temperature Rise around Bubble}

The previous section showed the bubble behavior and its effect on the elastic wall. In this section, we investigate how bubbles and laser heat transmits through the surrounding fluid affect the surroundings. As shown in Figure 3, a thermocouple was placed near the tip of the fiber in a wide space, and the temperature was measured during 10 seconds of laser irradiation. From the images taken at 
the same time, the bubble formation position was measured as the ratio $\gamma_{t}$ $\left(x_{t} / R_{\max }\right)$ of the distance from the center of the fiber to the thermocouple surface, $x_{t}$ and the maximum bubble radius, $R_{\max }$.

In the Case of $\gamma_{t}=0.5$ shown in Figure 6(a), after laser irradiation, the formed bubble grows, contacts the thermocouple at $\mathrm{A}$ at $t=0.10 \mathrm{~ms}$, and reaches the maximum diameter at $t=0.30 \mathrm{~ms}$. Thereafter, the bubble contracts while maintaining the state attached to the thermocouple, and collapses at $t=0.60 \mathrm{~ms}$. After the collapse, the rebounded bubbles are still attached to the thermocouple, and detaches from the thermocouple at section B at $t=0.74 \mathrm{~ms}$. In the case of $\gamma_{t}=$ 0.9 shown in Figure $6(\mathrm{~b})$, after laser irradiation, the growing bubble reaches its maximum diameter at the point $\mathrm{A}$ at $t=0.30 \mathrm{~ms}$ and contacts with the thermocouple. After that, the bubble begins to shrink, detaches from the thermocouple at part B at $t=0.54 \mathrm{~ms}$, and collapses at $t=0.60 \mathrm{~ms}$. The rebounded bubbles do not come into contact with the thermocouple.

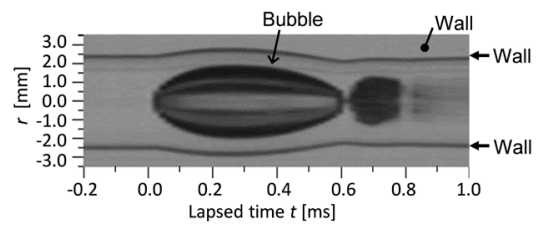

(a) $\gamma_{\mathrm{w}}=1.3, X_{\mathrm{w}}=2.5 \mathrm{~mm}$

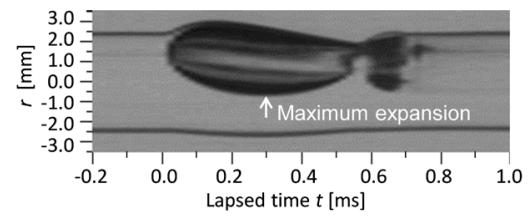

(b) $\gamma_{\mathrm{w}}=0.5, x_{\mathrm{w}}=1.0 \mathrm{~mm}$

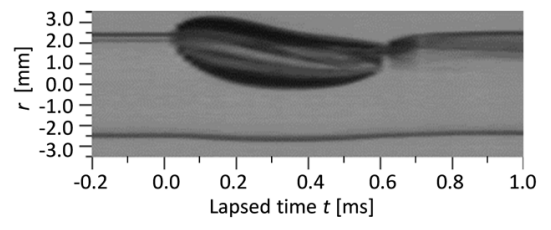

(c) $\gamma_{\mathrm{w}}=0.1, X_{\mathrm{w}}=0.3 \mathrm{~mm}$

Figure 5. Temporal change of a laser-induced bubble and an elastic wall. $T_{\mathrm{w}}=16^{\circ} \mathrm{C}-20^{\circ} \mathrm{C}, \beta=7.3 \mathrm{mg} / \mathrm{L}$.

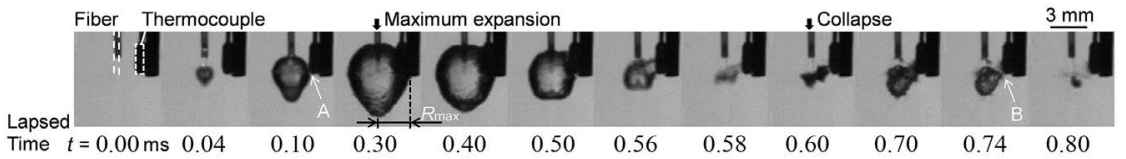

(a) $\gamma_{\mathrm{t}}=0.5, x_{\mathrm{t}}=1.0 \mathrm{~mm}$

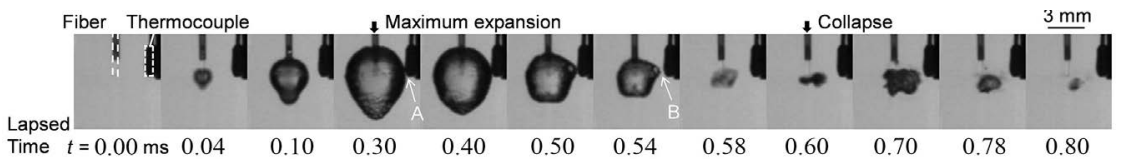

(b) $\gamma_{\mathrm{t}}=0.9, x_{\mathrm{t}}=2.0 \mathrm{~mm}$

Figure 6. Behaviors of a laser-induced bubble near a thermocouple. $T_{\mathrm{w}}=20^{\circ} \mathrm{C}-21^{\circ} \mathrm{C}$, $\beta=7.7-7.6 \mathrm{mg} / \mathrm{L}$. 
Figure 7 shows the measurement results of the temperature change around the bubble formed at the fiber tip when the laser pulse is irradiated for 10 seconds at a repetition frequency of $40 \mathrm{~Hz}$ (total laser heat load is estimated to be $400 \mathrm{~W}$ ) under the same conditions as in Figure 6. Figure 7(a) shows the temperature waveform measured with a thermocouple in the case of $\gamma_{t}=0.5\left(x_{t}=1.0\right.$ $\mathrm{mm})$. The temperature reaches a maximum of $46.3^{\circ} \mathrm{C}$ in about 1 second after the start of laser irradiation. After that, the laser is irradiated in a pulsed manner, but the temperature continues to be maintained at about $42^{\circ} \mathrm{C}-45^{\circ} \mathrm{C}$, indicating that the time-average temperature rise is captured. After laser irradiation, the temperature drops rapidly and returns to the original water temperature in about 1 second.

Figure $7(\mathrm{~b})$ shows the distribution of the temperature rise $\Delta T$ with respect to $\gamma_{t}$. Here, $\gamma_{t}<1.0$ indicates that the thermocouple enters the bubble during maximum expansion, and $\gamma_{t}=1.0$ indicates that the bubble contacts the thermocouple at maximum expansion. The ratio $t_{d} t_{i}$ of the time $t_{c}$ during which the bubble is in contact with the thermocouple during the laser irradiation time $t_{i}$ is also shown in the figure. From Figure 7(b), the temperature around the bubble and the contact time of the bubble increased with decreasing $\gamma_{t}$. In the case of $\gamma_{t}=1.8$ - 0.9 (when the bubble is separated from or almost in contact with the thermocouple), the temperature rise is about $5^{\circ} \mathrm{C}-10^{\circ} \mathrm{C}$. On the other hand, around $\gamma_{t}$ $=0.5-0.7$ (when the thermocouple enters the inside of the bubble), the temperature increases up to $35^{\circ} \mathrm{C}$ even if the contact time of the bubble is as short as several \%.

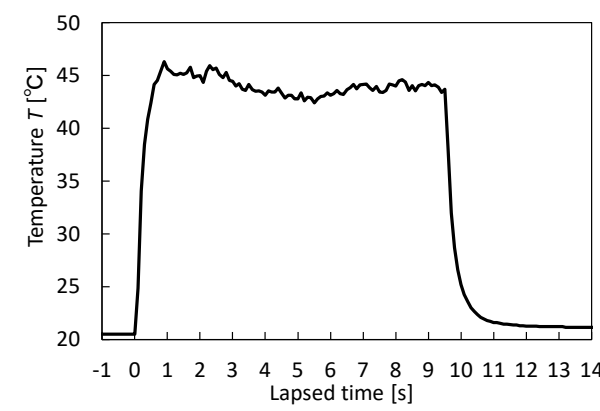

(a) Temporal change of temperature $\left(\gamma_{t}=0.5\right)$.

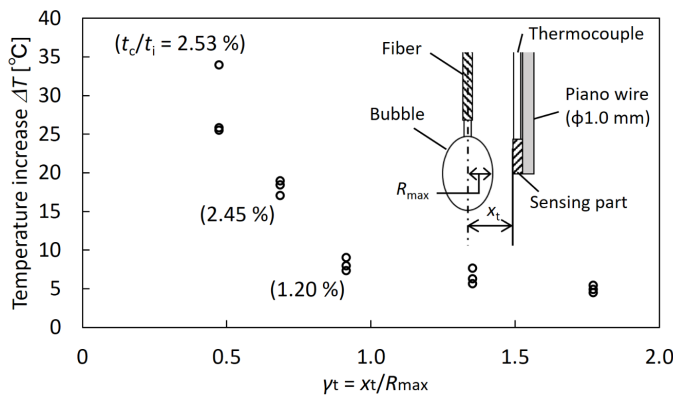

(b) Spatial distribution of temperature increase. $t_{i}$ : duration of pulsed laser irradiation $(10 \mathrm{sec}), t_{c}$ : total bubble contact time.

Figure 7. Temperature measurement near a laser-induced bubble. $T_{\mathrm{w}}=20^{\circ} \mathrm{C}-21^{\circ} \mathrm{C}, \beta=7.7-7.6 \mathrm{mg} / \mathrm{L}$. 


\section{Discussion}

In this study, some simplifications are made in simulating the situation of TUL treatment. In actual treatment, physiological saline is used as a perfusate for cooling and stone discharge, and it is assumed that the liquid quality is almost the same as the tap water used in this study. Furthermore, the elastic wall uses gelatin as a phantom of tissue, and simulates by focusing only on the softness and spatial scale of the elastic wall in this study. And further studies are needed on other parameters of biomaterials.

On the other hand, Brujan et al. [14] showed leaving a jet manner ejection of large deformation and the elastic wall material of the cell strain and elastic wall with the flow from the peripheral direction of the bubble through detail observations of bubbles formed near the flat elastic wall. Therefore, it can be pointed out the possibility of the damage of the elastic wall even in this study conducted under similar conditions, such as the bubble collapse caused by the bubbles formed near the elastic wall shown in Chapter 3.

In addition, Chan et al. [25] measured the temperature inside the bubble and confirmed that it rises from room temperature to about $65^{\circ} \mathrm{C}$. In this experiment shown in Chapter 4, it is predicted that the temperature can reach even higher instantaneously, considering the response speed of the thermocouple, short-time laser irradiation, and irradiation in a narrow space. The elastic wall deforms away from the bubble interface as shown previously, but it is also predicted that the temperature is high on the elastic wall when the bubble contacts the elastic wall. Therefore, when treatment is performed at the fiber position at $\gamma_{t}<1.0$, not only damage due to rapid deformation of living tissue due to bubble behavior but also exposure to high temperature for a short time due to contact between tissue and bubbles.

\section{Conclusions}

Observation of the behavior of bubbles and surrounding elastic walls in a narrow cylindrical space surrounded by soft material simulating the treatment environment at TUL, and measurement of the temperature around the bubbles were examined, and the following findings were obtained.

1) Bubbles formed in the narrow cylindrical space grow long in the axial direction of the cylindrical tube and collapse without showing a large splitting behavior.

2) As the bubble formation position moves from the center of the tube to the elastic wall side, the deformation of the elastic wall during bubble expansion increases. Bubbles formed near the elastic wall adhere to the elastic wall and collapse on the elastic wall.

3) The temperature around the bubble depends on the ratio of the bubble width to the fiber position, $\gamma_{t}$. The temperature rises about $5^{\circ} \mathrm{C}-10^{\circ} \mathrm{C}$ when $\gamma_{t}$ is about 1 or more, and rises about $35^{\circ} \mathrm{C}$ when $\gamma_{t}$ is less than 1 .

Based on the above, we present the risk of mechanical and thermal damage 
caused by laser irradiation in the vicinity of body tissue in TUL, and could show useful findings for safe treatment under various proposed laser irradiation conditions.

\section{Acknowledgements}

The authors thank Boston Scientific Japan co., ltd. for advice on medical viewpoint and providing a laser equipment. A part of this work was supported by JSPS KAKENHI Grant Number JP16K06092 and JP20K12610.

\section{Conflicts of Interest}

The authors declare no conflicts of interest regarding the publication of this paper.

\section{References}

[1] Ikeda, T., Yoshizawa, S., Tosaki, M., Allen, J.S., Takagi, S., Ohta, N., Kitamura, T. and Matsumoto, Y. (2006) Cloud Cavitation Control for Lithotripsy Using High Intensity Focused Ultrasound. Ultrasound in Medicine and Biology, 32, 1383-1397. https://doi.org/10.1016/j.ultrasmedbio.2006.05.010

[2] Maxwell, A.D., Cunitz, B.W., Kreider, W., Sapozhnikov, O.A., His, R.S., Harper, J.D., Bailey, M.R. and Sorensen, M.D. (2015) Fragmentation of Urinary Calculi in Vitro by Burst Wave Lithotripsy. The Journal of Urology, 193, 338-344. https://doi.org/10.1016/j.juro.2014.08.009

[3] Maeda, K., Maxwell, A.D., Kreider, W., Colonius, T. and Bailey, M.R. (2018) Investigation of the Energy Shielding of Kidney Stones by Cavitation Bubble Clouds during Burst Wave Lithotripsy. Proceedings of 10 th International Symposium on Cavitation, Baltimore, 14-16 May 2018, 626-630. https://doi.org/10.1115/1.861851 ch119

[4] Marks, A.J., Qiu, J., Milner, T.E., Chan, K.F. and Teichman, J.M.H. (2011) Laser Lithotripsy Physics. In: Rao, P.N., Preminger, M.G. and Kavanagh, P.J., Eds., Urinary Tract Stone Disease, Springer, Berlin, 301-309. https://doi.org/10.1007/978-1-84800-362-0 26

[5] Teichman, J.M.H., Rao, R.D., Glickman, R.D. and Harris, J.M. (1998) Holmium:YAG Percutaneous Nephrolithotomy: The Laser Incident Angle Matters. The Journal of Urology, 159, 690-694. https://doi.org/10.1016/S0022-5347(01)63701-7

[6] Schafer, S.A., Durville, F.M., Jassemnejad, B., Bartels, K.E. and Powell, R.C. (1994) Mechanisms of Biliary Stone Fragmentation Using the Ho:YAG Laser. IEEE Transaction on Biomedical Engineering, 41, 276-283. https://doi.org/10.1109/10.284946

[7] Zhong, P., Tong, H.L., Cocks, F.H., Pearle, M.S. and Preminger, G.M. (1998) Transient Cavitation and Acoustic Emission Produced by Different Laser Lithotripsy. The Journal of Endourology, 12, 371-379. https://doi.org/10.1089/end.1998.12.371

[8] Chan, K.F., Pfefer, T.J., Teichman, J.M.H. and Welch, A.J. (2001) A Perspective on Laser Lithotripsy: The Fragmentation Process. Journal of Endourology, 15, 257-273. https://doi.org/10.1089/089277901750161737

[9] Vassar, G.J., Chan, K.F., Teichman, J.M.H., Glickman, R.D., Weintraub, S.T., Pfefer, T.J. and Welch, A.J. (1999) Holmium YAG Lithotripsy Photothermal Mechanism. The Journal of Endourology, 13, 181-190. https://doi.org/10.1089/end.1999.13.181

[10] Frenz, M., Konz, F., Pratisto, H., Weber, H.P., Silenok, A.S. and Konov, V.I. (1998) 
Starting Mechanisms and Dynamics of Bubble Formation Induced by a Ho Yttrium Aluminum Garnet Laser in Water. Journal of Applied Physics, 84, 5905-5913. https://doi.org/10.1063/1.368906

[11] Lauterborn, W. and Bolle, H. (1975) Experimental Investigations of Cavitation-Bubble Collapse in the Neighborhood of a Solid Boundary. Journal of Fluid Mechanics, 72, 391-399. https://doi.org/10.1017/S0022112075003448

[12] Vogel, A., Lauterborn, W. and Timm, R. (1989) Optical and Acoustic Investigation of the Dynamics of Laser-Produced Cavitation Bubbles near a Solid Boundary. Journal of Fluid Mechanics, 206, 299-338. https://doi.org/10.1017/S0022112089002314

[13] Tomita, Y. and Shima, A. (1986) Mechanisms of Impulsive Pressure Generation and Damage Pit Formation by Bubble Collapse. Journal of Fluid Mechanics, 169, 535-564. https://doi.org/10.1017/S0022112086000745

[14] Brujan, E.A., Nahen, K., Schmidt, P. and Vogel, A. (2001) Dynamics of Laser-Induced Cavitation Bubbles near an Elastic Boundary. Journal of Fluid Mechanics, 433, 251-281. https://doi.org/10.1017/S0022112000003347

[15] Brujan, E.A., Nahen, K., Schmidt, P. and Vogel, A. (2001) Dynamics of Laser-Induced Cavitation Bubbles near Elastic Boundaries: Influence of the Elastic Modulus. Journal of Fluid Mechanics, 433, 283-314. https://doi.org/10.1017/S0022112000003335

[16] Kobayashi, K., Kodama, T. and Takahira, H. (2011) Shock Wave-Bubble Interaction near Soft and Rigid Boundaries during Lithotripsy: Numerical Analysis by the Improved Ghost Fluid Method. Physics in Medicine and Biology, 56, 6421-6440. https://doi.org/10.1088/0031-9155/56/19/016

[17] Kodama, T., Tomita, Y. and Shima, A. (1993) Interaction of a Bubble Attached to a Gelatine Wall with a Shock Wave: A Study of Tissue Damage Caused by Bubble Collapse. Transactions of the Japan Society of Mechanical Engineers. Series B, 59, 1431-1435. https://doi.org/10.1299/kikaib.59.1431

[18] Blake, J.R. and Gibson, D.C. (1981) Growth and Collapse of a Vapour Cavity near a Free Surface. Journal of Fluid Mechanics, 111, 123-140. https://doi.org/10.1017/S0022112081002322

[19] Chahine, G.L. (1977) Interaction between an Oscillating Bubble and a Free Surface. Journal of Fluid Engineering, 99, 709-716. https://doi.org/10.1115/1.3448889

[20] Ogasawara, T., Ito, S. and Takahira, H. (2018) The Growth and Collapse of a Bubble between Parallel Flat Free Surfaces. Proceedings of 10 th International Symposium on Cavitation, Baltimore, 14-16 May 2018, 1007-1012.

https://doi.org/10.1115/1.861851 ch192

[21] Ogasawara, T., Tsubota, N., Seki, H., Shigaki, Y. and Takahira, H. (2015) Experimental and Numerical Investigations of the Bubble Collapse at the Center between Rigid Walls. Journal of Physics. Conference Series, 656, Article ID: 012031. https://iopscience.iop.org/article/10.1088/1742-6596/656/1/012031 https://doi.org/10.1088/1742-6596/656/1/012031

[22] Sugimoto, Y., Yamanishi, Y., Sato, K. and Moriyama, M. (2015) Measurement of Bubble Behavior and Impact on Solid Wall Induced by Fiber-Holmium:YAG Laser. Journal of Flow Control, Measurement \& Visualization, 3, 135-143. https://doi.org/10.4236/ifcmv.2015.34013

[23] Sugimoto, Y., Nagata, D. and Sato, K. (2017) Fiber-Type-Laser Induced Bubble Behaviors in Narrow Space with a Soft Wall. Proceedings of 10th International Symposium on Measurement Techniques for Multiphase Flow, Hong Kong, ISMTMF-R001-046. 
[24] Sugimoto, Y., Nagata, D. and Sato, K. (2018) Behavior of Bubble Induced by Fiber-Type Laser for TUL near Soft Wall with Deformability. Proceedings of 10 th International Symposium on Cavitation, Baltimore, 14-16 May 2018, 135-139. https://doi.org/10.1115/1.861851 ch27

[25] Chan, K.F., Pfefer, T.J., Hammer, D.X., Jansen, E.D., Frenz, M. and Welch, A.J. (1998) Fluorescence-Based Temperature Measurement in Laser-Induced Vapor Bubbles. Proceedings of SPIE-The International Society for Optical Engineering, 3254, 276-286. https://doi.org/10.1117/12.308174 